\title{
Developmental Disturbance of Dentin with Probable Diagnosis of Dentin Dys- plasia Type 1d: A Rare Case Report
}

\section{A Rashidian', Sh Zahraei ${ }^{2}$, SS Hashemi}

1-Assistant professor, Pediatric dentistry Dept, Faculty of Dentistry, Tehran Medical Sciences, Islamic Azad University, Tehran, Iran 2-Postgraduate student,Pediatric dentistry Dept, Faculty of Dentistry, Tehran Medical Sciences, Islamic Azad University, Tehran, Iran 3-Dentist

\begin{tabular}{l}
\hline ARTICLE INFO \\
Article History \\
Received: Nov 2018 \\
Accepted: Dec 2018 \\
ePublished: Jan 2019 \\
\hline
\end{tabular}

Corresponding author: postgraduate student, Pediatric dentistry Dept, Faculty of Dentistry, Tehran Medical Sciences, Islamic Azad University, Tehran, Iran,shohrehz@, gmail.com

\begin{abstract}
Background and Aim: Dentin dysplasia (DD) is a rare disturbance of dentin formation, characterized by normal enamel but atypical dentin formation with abnormal pulpal morphology. In DD type I, the teeth appear clinically normal in morphologic appearance and color. Radiographic analysis shows obliteration of all pulp chambers as well as short, blunted, and malformed or absent roots with multiple periapical radiolucencies involving apparently intact teeth.

Case Presentation: We present a case of a 15-year-old girl with a developmental disturbance of dentin similar to DD type 1. Radiographic examination revealed multiple affected teeth with unique findings in accordance with DD 1d subtype. This article highlights the clinical and radiographic findings of this condition along with the differential diagnosis and management.
\end{abstract}

Conclusion: Management of patients with DD is difficult and requires a multidisciplinary approach. Early diagnosis of the condition is important for the initiation of effective preventive treatment.

Keywords: Tooth Abnormalities, Dentin Dysplasia, Rootless Teeth

J Res Dent maxillofac Sci 2019;4(2):40-44.

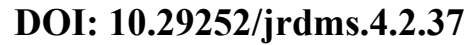

\section{Introduction:}

Dentin dysplasia (DD) is a rare hereditary autosomal dominant disorder with unknown etiology, which affects approximately one patient in every 100,000 cases. ${ }^{(1)}$ There seems to be no sex predilection. $^{(1)}$
It is characterized by normal enamel but altered dentin structure with abnormal pulpal morphology affecting either the primary or both the primary and permanent dentitions. ${ }^{(2)}$ The condition was first described in 1922 by Ballschmiede. ${ }^{(3)}$ 
Rushton used the term "dentinal dysplasia" for the first time. ${ }^{(4)}$ Generally, two main classes of DD are recognized based on the clinical and radiographic appearance, including type 1 (radicular DD) and type 2 (coronal DD). These terms were first described by Witkop to indicate the dental parts that are primarily involved. ${ }^{(5)}$ DD type 1 is characterized by normal or slightly amber-colored dental crowns with no or primitive root development, abnormal growth of dentin in pulp chambers with reduced pulp space in permanent teeth, incomplete or total obliteration of pulp chambers, and periapical radiolucencies or cysts, resulting in premature loss of teeth. ${ }^{(6)}$

DD type 2 is characterized by yellow, brown, grey-amber, translucent primary teeth with complete pulpal obliteration. The permanent teeth have a normal appearance or might be slightly amber-colored. The permanent dentition is clinically normal but highly susceptible to attrition because of abnormal dentin. Roots are normal in size and shape with a thistle tubeshaped pulp chamber containing pulp stones..$^{(7)}$

The purpose of this article was to present a case with a rare disorder, i.e. DD type 1, with an overview of its diagnosis and management.

\section{Case Presentation:}

A 15-year-old female patient presented to the Pediatric Dentistry Department of the Dental Faculty of Islamic Azad University of Medical Sciences, Tehran, Iran, with the chief complaint of abscess of upper teeth. Her past medical and dental history was noncontributory to the diagnosis. The extraoral examination was negative for any abnormal findings (Figure 1).

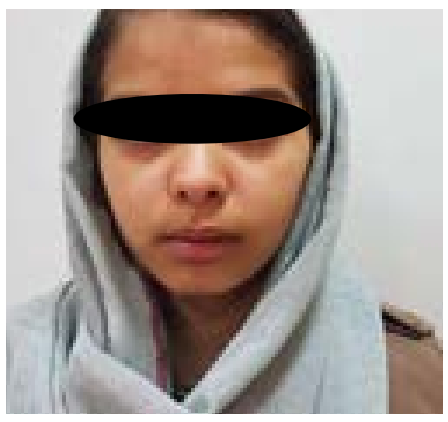

Figure 1: Extraoral view.
The intraoral examination revealed increased overjet and $2 \mathrm{~mm}$ deviation of the midline to the right. The crowns appeared normal in color and shape, and the teeth had no mobility

(Figure 2).

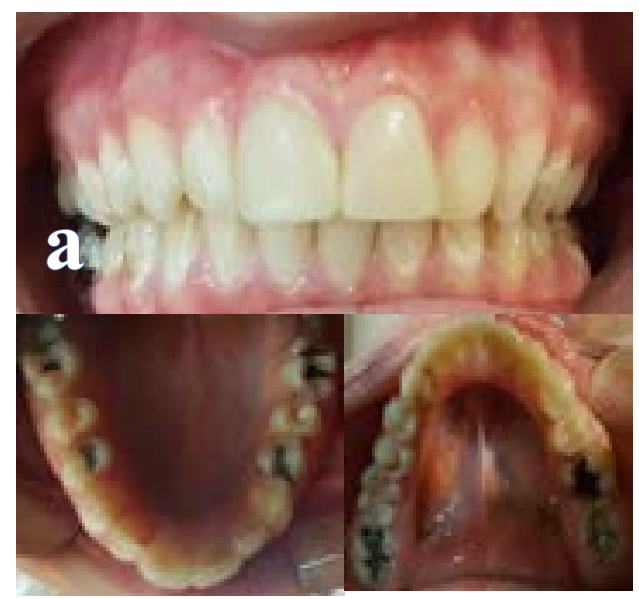

Figure 2- Intraoral views: (a) Frontal.

(b) Maxillary arch. (c) Mandibular arch.

Radiographic examinations revealed adequate enamel thickness on the cusp tips of all the teeth. Several teeth had short, pointed roots and obliterated pulp chambers. Pulp stones were found in teeth \#35 and \#45. A Well-defined periapical radiolucency was present in association with the apex of tooth \#11 (Figure 3).

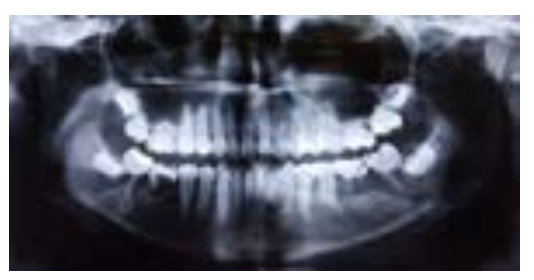

Figure 3: Panoramic radiograph showing pulp stones and shortened roots.

The patient's medical history revealed no systemic disease. Clinical and radiograph examinations of the patient's parents and siblings revealed no such disorder or any previous familial history of such condition. Also, there was no history of complications in the primary dentition, such as premature loss or abnormal shape or color. 
Therefore, on the basis of the clinical and radiographic appearance, a diagnosis of DD type I was suspected. Accordingly, the patient was considered as a first-generation sufferer of DD in her family.

Considering the age of the patient, treatment was aimed at maintaining the health of the existing teeth. A multidisciplinary approach was planned for the patient. She was referred to the Endodontics Department for treatment of the upper teeth. Subsequently, teeth \#11 and \#21 were diagnosed with a necrotic pulp after performing vitality tests due to composite marginal leakage. Since tooth \#36 showed no mobility or furcation involvement, it was restored with a stainless steel crown (SSC) (Figure 4), while other decayed teeth were restored with composite restorations.

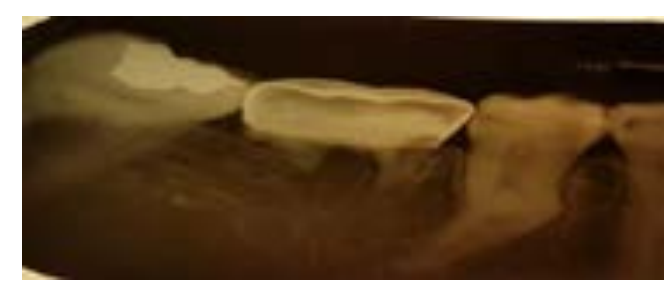

Figure 4: Stainless steel crown (SSC) placement on tooth \#36. Note the pulp stone and the associated root bulging in tooth \#35.

The patient decided to preserve tooth \#46 since it was asymptomatic, and extraction and replacement with an implant-supported prosthesis were advised once her growth is complete. The importance of good oral hygiene and regular follow-up visits was emphasized.

\section{Discussion:}

DD type I (radicular DD; rootless teeth) is a rare hereditary autosomal dominant disturbance of dentin formation characterized by a defective dentin development with clinically normal appearing crowns in both primary and permanent dentitions. (8) Although the molecular source of DD type I has remained unclear, Xiong et al reported that a missense mutation in SSUH2 gene on 3p26.1-3p24.3 in association with other genes that affect odontogenesis may have a role in the develop- ment of dysplastic teeth. ${ }^{(9)}$ Radiographic analysis shows obliteration of pulp chambers, short, restrained, and malformed or absent roots, and multiple periapical radiolucencies associated with non-carious teeth. ${ }^{(10)}$ DD type I has been referred to as "rootless teeth" because of the shortened root length due to loss of organization of radicular dentin. ${ }^{(8)}$

The etiology of DD is still unknown; however, several theories have been proposed in the dental literature. Logan et al proposed that the dentinal papilla is responsible for the abnormalities in root development. ${ }^{(11)}$ In a scanning electron microscopic (SEM) study, Sauk et al postulated that an invagination of the epithelial root sheath is responsible for abnormal dentin formation. ${ }^{(12)}$ Wesley et al disagreed with this suggestion and offered an alternative hypothesis. ${ }^{(13)}$ They stated that DD type 1 results from erroneous interaction of odontoblasts with the ameloblastic layer, leading to abnormal differentiation and/or function of these odontoblasts. ${ }^{(13)}$

Clearly, the precise nature of the defect has yet to be determined. The cause of periapical radiolucencies in DD type I has not yet been identified. Steidler et al suggest that they are the result of pulpal necrosis, occurring either secondary to caries or spontaneously. ${ }^{(14)}$ In the present case, the periapical radiolucency was caused by the necrotic pulp of tooth \#11 secondary to a defective composite restoration.

According to O'Carroll et al, DD type 1 is subdivided into four subtypes (Table 1).

Table 1: Carroll's classification of dentin dysplasia type 1 (DD1)

\begin{tabular}{cl}
\hline Dentin Dysplasia & \multicolumn{1}{c}{ Clinical and Radiographic Appearance } \\
\hline Type 1 (DD1) & $\begin{array}{l}\text { Primary and permanent teeth with normal } \\
\text { crowns (color and morphology), shortened } \\
\text { roots, periapical radiolucencies, and pulp } \\
\text { obliteration }\end{array}$ \\
\hline DD1a & $\begin{array}{l}\text { Complete obliteration of pulp chambers and } \\
\text { no root development with several periapical } \\
\text { radiolucent areas }\end{array}$ \\
\hline DD1b & $\begin{array}{l}\text { Horizontal, crescent-shaped, radiolucent } \\
\text { pulpal remnants and few millimeters of root } \\
\text { development with several periapical } \\
\text { radiolucent areas }\end{array}$ \\
\hline DD1c & $\begin{array}{l}\text { Two horizontal, crescent-shaped, } \\
\text { radiolucent lines and significant but } \\
\text { incomplete root development, with or } \\
\text { without periapical radiolucent areas }\end{array}$ \\
\hline DD1d & $\begin{array}{l}\text { Visible pulp chambers and oval pulp stones } \\
\text { in the coronal third of root canal, with } \\
\text { bulging of the root around the stone, and } \\
\text { few, if any, periapical radiolucent areas }\end{array}$ \\
\hline
\end{tabular}


$(15,16)$ Variations in root and pulp formation are due to the disparities in the timing of dentinal disorganization. With early disorganization, roots are extremely short or absent with no pulp. With later disorganization, roots are shortened with crescent-shaped or chevronshaped pulp chambers. With late disorganization, typical root lengths exist with pulp stones present in a normal-shaped pulp chamber. This variability is most intense in the permanent dentition and can vary for each person and from tooth to tooth. ${ }^{(17)}$

The findings related to the present patient were the same as those of DD type $1 \mathrm{~d}$, including the bulging of the coronal third of the roots with pulp stones and shortened roots of molars; this type is the least severe form of DD. ${ }^{(16)}$

DD type I should be differentiated from other conditions, such as dentinogenesis imperfecta and DD type 2. Dentinogenesis imperfecta have a DD-like phenotype but it is more similar to DD type 2. Normal-appearing crowns, shortened roots, and association of radiolucencies with non-carious teeth are important characteristics of DD type 1 . In dentinogenesis imperfecta and DD type 2 , teeth may show color changes, hypomineralized and exposed dentin, bulbous crowns, cervical constriction, thick and short roots, and obliterated pulp chambers. ${ }^{(1)}$

Teeth with radiographic or histological features of DD can be associated with a number of disorders, such as calcinosis, osteogenesis imperfecta, Ehlers-Danlos syndrome, Goldblatt syndrome, Schimke immuno-osseous dysplasia, and brachioskeletogenital syndrome. ${ }^{(8)}$ With regard to our case, the findings did not support any association with systemic disorders. No hereditary pattern was recognized after examining the panoramic radiographs of the patient's family members. It would have been better if we could study the histopathological slices of the teeth but this was not possible since the patient wanted to preserve the teeth. Also, genetic evaluation might have been helpful.

Management of patients with DD is difficult and requires a multidisciplinary approach. The goal of treatment is to maintain the teeth for as long as possible. Follow-up and routine conservative treatment are often advised. Maintenance of periodontal health through adherence to oral hygiene instructions is vital to the survival of mobile teeth. ${ }^{(18)}$ Endodontic treatment is contraindicated in teeth with total obliteration of root canals and pulp chambers. ${ }^{(8)}$ Orthodontic treatment can be performed; however, further resorption of the roots, loosening of the teeth, and premature exfoliation may occur due to the resistance of short roots to orthodontic forces. ${ }^{(2)}$ Prosthetic rehabilitation, including the use of dentures, overdentures, and dental implants, may be required. However, treatment varies according to the age of the patient, the severity of the problem, and the presenting complaint. ${ }^{(6)}$

\section{Conclusion:}

DD type 1 is a rare inherited abnormality of the dentin that leads to premature exfoliation of the primary and permanent teeth. Early diagnosis of the condition is important for the initiation of effective preventive treatment. Since many cases of DD type I may be primarily asymptomatic, these malformations might easily be overlooked without radiographic examination. In this regard, pediatric dentists have an important role in the early diagnosis of this disorder and in guiding the patients regarding the selection of measures to prolong the maintenance of the affected teeth.

\section{References:}

1. de La Dure-Molla M, Philippe Fournier B, Berdal A. Isolated dentinogenesis imperfecta and dentin dysplasia: revision of the classification. Eur J Hum Genet. 2015 Apr;23(4):445-51.

2. Malik S, Gupta S, Wadhwan V, Suhasini GP. Dentin dysplasia type 1 - A rare entity. J Oral Maxillofac Pathol. 2015 Jan-Apr;19(1):110.

3. Chamberlain BB, Hayward JR. Management of dentin dysplasia and facial disharmony. Spec Care Dentist. 1983 May-Jun;3(3):113-6.

4. Rushton MA. A case of dentin dysplasia. Guys Hosp Rep. 1939;89:369-73.

5. Witkop CJ Jr. Hereditary defects of dentin. Dent Clin North Am. 1975 Jan;19(1):25-45.

6. Fulari SG, Tambake DP. Rootless teeth: Dentin dysplasia type 1. Contemp Clin Dent. 2013 OctDec;4(4):520-522.

7. Daryani D, Nair GR, Naidu G. Dentin dysplasia type II: An exclusive report of two cases in siblings. J Indian Acad Oral Med Radiol. 
2017;29:132-4.

8. Sekerci AE, Etoz M, Sahman H, Sisman Y, Nazlim S. A rare condition affecting the primary and permanent dentition: Dentin dysplasia type I. J Oral Maxillofac Radiol. 2013;1(1):13-16.

9. Xiong F, Ji Z, Liu Y, Zhang Y, Hu L, Yang Q, et al. Mutation in SSUH2 Causes Autosomal-Dominant Dentin Dysplasia Type I. Hum Mutat. 2017 Jan;38(1):95-104.

10. Carmem DVSdM, Lúcia dFAdDM, Heloísa CSS, Markelane SS, Graciela MOS, Marina dDMdL. Dentin Dysplasia Type I-Diagnosis and Treatment: Case Report. Adv Dent \& Oral Health. 2017 Oct;6(1):555676.

11. Logan J, Becks H, Silverman S Jr, Pinnborg JJ. Dentinal dysplasia. Oral Surg Oral Med Oral Pathol. 1962 Mar;15:317-33.

12. Sauk JJ Jr, Lyon HW, Trowbridge HO, Witkop CJ Jr. An electron optic analysis and explanation for the etiology of dentinal dysplasia. Oral Surg Oral Med Oral Pathol. 1972 May;33(5):763-71.

13. Wesley RK, Wysocki GP, Mintz SM, Jackson J. Dentin dysplasia type I. Clinical, morphologic, and genetic studies of a case. Oral Surg Oral Med Oral Pathol. 1976 Apr;41(4):516-24.

14. Steidler NE, Radden BG, Reade PC. Dentinal dysplasia: A clinicopathological study of eight cases and review of the literature. Br J Oral Maxillofac Surg. 1984 Aug;22(4):274-86.

15. O'Carroll MK, Duncan WK, Perkins TM. Dentin dysplasia: review of the literature and a proposed subclassification based on radiographic findings. Oral Surg Oral Med Oral Pathol. 1991 Jul;72(1):119-25.

16. Sahoo SR, Aggarwal S. Dentin dysplasia type 1d: A rare case. Indian J Dent Res. 2014 NovDec;25(6):832-4.

17. American Academy of Pediatric Dentistry. Guideline on dental management of heritable dental developmental anomalies. Pediatr Dent. 2013 Sep-Oct;35(5):E179-84.

18. Khandelwal S, Gupta D, Likhyani L. A Case of Dentin Dysplasia with Full Mouth Rehabilitation: A 3-year Longitudinal Study. Int J Clin Pediatr Dent. 2014 May-Aug;7(2):119-124.
Please cite this paper as:

Rashidian A, Zahraei S, Hashemi S. Developmental Disturbance of Dentin with Probable Diagnosis of Dentin Dysplasia Type 1d: A rare case report . J Res Dentomaxillofac Sci. 2019; 4 (2):4044. 